\title{
The Economic Burden of Bleeds and Transfusions in Selected Surgeries: A Retrospective Multi Center Analysis from the US Perspective
}

\author{
Ramirez Manuel $\mathrm{G}^{1 *}$, Castillo George $\mathrm{F}^{1}$ and Ramirez Manuel $\mathrm{A}^{2}$ \\ ${ }^{1}$ Global Advanced Surgery Clinical, North America \\ ${ }^{2}$ Baxter Healthcare, Mexico
}

*Corresponding author: Ramirez Manuel G, Global Advanced Surgery Clinical/HEOR, Advanced Surgery, Baxter Healthcare Corporation, 1 Baxter Way, Deerfield, IL 60015, USA.

To Cite This Article: : Ramirez Manuel G. The Economic Burden of Bleeds and Transfusions in Selected Surgeries: A Retrospective Multi Center Analysis from the Us Perspective. Am J Biomed Sci \& Res. 2019 - 2(5). AJBSR.MS.ID.000610. DOI: 10.34297/AJBSR.2019.02.000610

Received: March 26, 2019 | Published: May 02, 2019

\begin{abstract}
Objective: To identify the economic impact of bleeds and transfusions by analyzing the effect of hemostatic agents (active and passive) on adults undergoing Cardio Vascular/Thoracic \& Ortho-Ortho Spine surgeries in US hospitals.

Methods: A Retrospective review of intraoperative data from 72 Cardio Vascular/Thoracic, and 39 Ortho-Ortho Spine surgeries performed between August-December 2018, from different US Urban Hospitals was performed to determine the economic impact of bleeding and transfusions associated with the utilization efficiency of active or passive hemostatic agents.

Results: Of the 111 cases analyzed, 157 hemostatic agents were used. Utilization efficiency (a percentage defined as the amount of product used divided by the amount opened) was calculated. Utilization efficiency or products such as Flowable were $84 \%$ and Sealants $97 \%$, Products with medium utilization efficiency such as Gelatin Powder were $76 \%$ and Oxidized Cellulose $72 \%$, and products with a low utilization efficiency such as Thrombin were $46 \%$, and Gelatin Sponge \& Bone Wax 42\%. The average estimated blood loss for all surgeries was $455 \mathrm{~mL}$. The total blood product units used included Packed Red Blood Cells (PRBC) 12U, Fresh Frozen Plasma (FFP) and Platelets 10U, and Cryoprecipitate 52U. The economic impact of bleeding and transfusions ranged from $\$ 9,824$ to $\$ 29,296$ depending on the surgery and the hemostatic agent used.
\end{abstract}

Conclusions: Surgical bleeds and transfusions can have substantial financial and medical consequences. Using appropriate hemostatic agents intraoperatively may potentially reduce bleeds requiring transfusion and likely improve patient post-surgical outcomes. The use of active hemostatic agents resulted in a substantial Blood Product Use savings of \$19,472 (50\%) per patient. The use of active hemostatic agents may result in effective and cost-beneficial outcomes compared with passive hemostatic agents.

Clinical relevance: The use of Active Hemostat Agents may result in better clinical and possibly economic (bleeding and transfusion) outcomes in Cardio Vascular/Thoracic \& Ortho-Ortho Spine surgeries.

Keywords: Hemostatic agents; Active and passive; LUR; Bleeds; Transfusions

\section{Introduction}

Bleeding is a complication of surgery that may lead to substantial morbidity and mortality. In different type of surgeries (CV, Vascular, Thoracic, Neuro/Neuro-Spine), severe bleeding occurs in approximately $7 \%$ of cases [1] and is associated with an increased risk of post-operative mortality [2]. The burden of bleeding in the United States is costly for patients and for the healthcare system. Patients with uncontrolled intraoperative bleeding can be four times more likely to die from a surgical procedure [3]. These patients receive more blood transfusions, have longer hospital stays and have statistically worse outcomes, including significantly higher morbidity rates. The American Medical Association and the Joint Commission have identified blood transfusions as one of the top five over-utilized therapeutic procedures in the U.S. [4]. Efficient surgeries with limited bleeding may be one potential solution. Notable National Health Organizations have begun to address the issue of how to ensure more efficient utilization of blood transfusions.

Bleeding is an anticipated consequence of virtually all types of surgical procedures. Ideally, every surgical procedure concludes with hemostasis of the surgical field and an optimal patient outcome. The rates of bleeding and bleeding-related complications ranged from $7.5 \%$ with reproductive organ procedures to 
$47.4 \%$ with cardiac procedures [3]. Blood transfusions are a common practice in hospitals around the world, where up to $20 \%$ of patients who undergone surgery and $35 \%$ of patients in intensive care units receive at least one blood transfusion $[5,6]$. Depending on the bleeding type and the quantity of blood loss, this can lead to substantial morbidity and mortality. The rates of bleeding and bleeding-related complications (hemorrhage, anemia, thrombocytopenia, hematoma, embolic events or venous thrombosis) ranged from $7.5 \%$ with reproductive organ procedures to $47.4 \%$ with cardiac procedures [7]. Using Blood Products in those high-risk bleeding surgeries can lead to costs ranging from $\$ 2,805-\$ 17,279$ for reproductive organ and spinal. Patients with bleeding-related complications and/or blood product transfusions experienced longer hospital stay (4.9-18.7 vs. 2.6-9.1 mean days) and spent more time in the ICU (0.2-8.9 vs. 0.04-1.2 mean days) compared to patients without these events [8].

Patients who receive blood transfusions have a substantially higher risk of adverse events, resulting in up to three times longer hospital stays compared to patients who do not receive transfusions. Furthermore, blood transfusions are expensive, given not only the cost of the product, but also the substantial expense of labor associated with blood management. Although necessary in terms of care and its quality, these transfusions involve significant costs. In 2008, four different studies were carried out in the United States which concluded that the costs of blood bags alone varied between $\$ 332$ and $\$ 717[9,10]$.

The complexity of surgical procedures, where blood loss can have devastating consequences, creates challenges for a hospital's resources. If not well managed, intraoperative bleeding can threaten patient near-term and long-term outcomes and expend unnecessary hospital resources. Blood transfusions have become an increasingly frequent practice in U.S. hospitals. In fact, between 1997 and 2011, blood transfusions increased 134 percent. Current estimates show that as many as 40 to 50 percent of blood transfusions may be unnecessary, potentially putting patients' lives in jeopardy, and could be costing individual hospitals as much as $\$ 1$ million per year $[11,12]$. In addition to the supply limitations on blood and the costs associated with transfusions, transfusions may lead to suboptimal patient outcomes. Blood transfusions are frequently associated with longer patient length of stay, increased mortality and morbidity, in addition to increased costs and resource utilization. Reducing the need for blood transfusions by reducing blood loss during surgery, is one solution to eliminate wasteful utilization of resources, likely improve patient post-surgical outcomes and reduce costs to the hospital.

Blood conservation strategies are associated with nearly half the perioperative morbidity of conventional management $(39.1 \%$ vs. $23.5 \%, \mathrm{p}=0.046$ ), as assessed by a composite of any of 10 major postoperative complications [13]. The implementation of new methods, supported by innovations in medical technology that allow for the continuous measurement of hemoglobin values, would facilitate decision-making regarding transfusions, allowing transfusions where medically necessary and at the right moment, therefore reducing unnecessary transfusions [14].

\section{Methods}

A Retrospective analysis intraoperative data for 72 Cardio Vascular/Thoracic procedures and 39 Ortho-Ortho Spine surgeries performed between August and December 2018, from different US Urban Hospitals, whereby, the quantity of bleeding and transfusions associated with the utilization efficiency of active or passive hemostat products was analyzed to determine the economic impact of bleeds and transfusions.

\section{Data Source}

Source: Own elaboration with data from US Vital Edge Program, LUR Analyses retrospective analysis.

Data for this retrospective analysis was obtained from the Live Utilization Review (LUR) US Vitale Edge Data Base Program, a repository of product utilization efficiency, estimated blood loss (EBL), economic and resource use data developed for quality and utilization benchmarking purposes.

The database includes National US representative hospital data based on bed size, geographic region, and teaching hospital status. The database comprises data from 111 cases analyzed (72 Cardio Vascular/Thoracic \& 39 Ortho-Ortho Spine surgeries) and 157 hemostatic agents from August-December 2018.

\section{Database Case Selection Criteria}

The records of the Cardio Vascular/Thoracic \& Ortho-Ortho Spine surgeries included in this retrospective of prospectively collected intraoperative database analysis were of patients that underwent elective or urgent surgeries performed between AugustDecember 2018, from different US Urban Hospitals.

\section{Data Extracted for Analyses}

Data regarding surgery types [(Cardio Vascular/Thoracic \& Ortho-Ortho Spine), hemostatic agents (active and passive)], energy device, EBL, cell saver used, total blood product units used (packed red blood cells (PRBC), fresh frozen plasma (FFP), platelets and cryoprecipitate) were extracted. Hemostatic agent (active and passive) utilization efficiency was calculated as a percentage defined as the amount of product used divided by the amount opened (Table 1).

\section{Statistical Analyses}

Hypothesis tests were not made, derived from the conformation on the observational data, full inhibition of bleeding was not assessed with the setup of active/passive hemostatic agents, correlation matrix and linear regression was performed in order to represent the effect of the usage of active and passive hemostatic in the EBL, linear regression showed that the use of active hemostatic agents improve 5 more times the reduction of blood lost $(\mathrm{mL})$.

\section{Methodology}

\section{Cost Analyses}

Cost analysis was performed by examining the cost (system cost) of using Blood Products (RBC, Platelets, FFP and Cryoprecipitates) from the Society for the Advancement of Blood 
Management 2018 and Transfusions and Their Costs: Managing Patients Needs and Hospitals Economics, J Basha, R Dewitt, D Cable, G Jones. Transfusions and Their Costs: Managing Patients Needs and Hospitals Economics. The Internet Journal of Emergency and Intensive Care Medicine 2005. The first data entered the matrix were the ratio of units transfused per patient depending on the type of surgeries analyzed, the EBL and the cost of Blood Products used per surgery type and hemostatic product used (active or passive).
To create the cost-benefit matrix, the savings obtained from the number of units transfused per surgery type were considered. These savings were classified by different types of surgeries identified by the ratio of units transfused. The total cost of Blood Products used was analysed with the EBL per surgery and hemostatic product (active or passive) used versus those without EBL per surgery and hemostatic product (active or passive used).

\begin{tabular}{|c|c|c|c|c|c|c|c|c|c|c|}
\hline Product & \# Pkgs & $\begin{array}{c}\text { All } \\
\text { Cases - \% } \\
\text { Efficiency }\end{array}$ & \# Pkgs & $\begin{array}{l}\text { CV - } \% \\
\text { Efficiency }\end{array}$ & \# Pkgs & $\begin{array}{l}\text { Vascular - \% } \\
\text { Efficiency }\end{array}$ & \# Pkgs & $\begin{array}{c}\text { Thoracic } \\
-\quad \% \\
\text { Efficiency }\end{array}$ & \# Pkgs & $\begin{array}{l}\text { Ortho/Ortho } \\
\text { Spine - \% } \\
\text { Efficiency }\end{array}$ \\
\hline Surgifoam 50 Sponge & 36 & $51 \%$ & 17 & $76 \%$ & 7 & $51 \%$ & - & - & 12 & $27 \%$ \\
\hline Gelfoam - sponge 100 & 12 & $26 \%$ & - & - & 9 & $52 \%$ & - & - & 3 & $0 \%$ \\
\hline Surgifoam - powder 1gm & 2 & $60 \%$ & - & - & & & - & - & 2 & $60 \%$ \\
\hline Thrombin (Bovine 5000 IU) & 16 & $59 \%$ & 8 & $45 \%$ & 1 & $100 \%$ & - & - & 7 & $32 \%$ \\
\hline $\begin{array}{l}\text { Thrombin (Recombinant } \\
5000 \mathrm{IU})\end{array}$ & 12 & $43 \%$ & - & - & 9 & $51 \%$ & - & - & 3 & $35 \%$ \\
\hline FloSeal 5mL & 8 & $53 \%$ & 4 & $100 \%$ & 1 & $75 \%$ & - & - & 3 & $100 \%$ \\
\hline FloSeal $10 \mathrm{~mL}$ & 11 & $53 \%$ & 6 & $67 \%$ & - & - & - & - & 5 & $39 \%$ \\
\hline Tisseel 4mL & 2 & $100 \%$ & 2 & $100 \%$ & - & - & - & - & - & - \\
\hline Surgiflo KIT (w/Thrombin) & 9 & $66 \%$ & - & - & 1 & $100 \%$ & - & - & 8 & $31 \%$ \\
\hline Coseal 8mL & 7 & $77 \%$ & - & - & 7 & $77 \%$ & - & - & & \\
\hline Tachosil $4.8 \times 4.8$ & 3 & $87 \%$ & - & - & 3 & $87 \%$ & - & - & & \\
\hline Duraseal 3mL & 2 & $100 \%$ & - & - & & & - & - & 2 & $100 \%$ \\
\hline Surgicel Fibrillar $4 \mathrm{x} 4$ & 17 & $62 \%$ & 12 & $83 \%$ & 5 & $40 \%$ & & & - & - \\
\hline Surgicel Original 2x14 & 19 & $38 \%$ & 4 & $13 \%$ & 12 & $50 \%$ & 3 & $50 \%$ & - & - \\
\hline Surgicel-Nu-kknit 4x8 & 5 & $100 \%$ & 5 & $100 \%$ & - & - & - & - & - & - \\
\hline Instat MCH 1gm & 1 & $0 \%$ & 1 & $0 \%$ & - & - & - & - & - & - \\
\hline Bone Wax 2.5g & 26 & $40 \%$ & 14 & $67 \%$ & 1 & $0 \%$ & - & - & 11 & $54 \%$ \\
\hline
\end{tabular}

Source: Own elaboration with data from US Vital Edge Program, LUR Analyses retrospective analysis.

\section{Model analyses}

This model estimated the results from a retrospective analysis of prospectively collected intraoperative data (72 Cardio Vascular/ Thoracic \& 39 Ortho-Ortho Spine surgeries), performed between August-December 2018, from different US Urban Hospitals; the quantity of bleeding and transfusions associated with the utilization efficiency of active or passive hemostat products was analyzed to determine the economic impact of bleeds and transfusions.The cost analysis was performed by examining the cost (system cost) of using Blood Products (RBC, Platelets, FFP and Cryoprecipitates.

Results

Table 2: Estimated Blood Loss and Cell Salvage Used.

\begin{tabular}{|c|c|c|c|c|}
\hline Category & CV & Vascular & Thoracic & Ortho/Ortho-Spine \\
\hline Number of Cases & 37 & 15 & 9 & 37 \\
\hline Percentage Re-do Cases & $8 \%$ & $27 \%$ & 4.02 & 358 \\
\hline Average EBL (mL) & 631 & 479 & $0 / 0 / 0 / 0$ & $0 / 0 / 0 / 0$ \\
\hline $\begin{array}{c}\text { Blood Transfused PRBC/F F } \\
\text { P/P It/Cryo/Auto }\end{array}$ & $11 / 10 / 10 / 52$ & $4 / 0 / 0 / 0$ & $0(0 \%)$ & $0(0 \%)$ \\
\hline $\begin{array}{c}\#(\%) \text { of cases requiring any } \\
\text { blood transfusion }\end{array}$ & $5(13.51) \%$ & $0(0 \%)$ & $0(0 \%)$ & $2(5.40 \%)$ \\
\hline $\begin{array}{c}\#(\%) \text { of cases using cell } \\
\text { salvage }\end{array}$ & $32(86.48 \%)$ & $2(13.33 \%)$ & \\
\hline
\end{tabular}

Of the 111 cases analyzed, 157 hemostatic agents were used; utilization efficiency (a percentage defined as the amount of product

used divided by the amount opened). Utilization efficiency for products such as Flowable $84 \%$ and Sealants $97 \%$. Products with 
medium utilization efficiency included Gelatin Powder76\% and Oxidized Cellulose $72 \%$. Products with a low utilization efficiency included Thrombin 46\%, Gelatin Sponge \& Bone Wax 42\% (Figure 1). The average estimated blood loss for all surgeries was $455 \mathrm{~mL}$ and the total blood product units used included Packed Red Blood Cells (PRBC) 12U, Fresh Frozen Plasma (FFP) and Platelets 10U, and Cryoprecipitate 52U. The average EBL for CV was $631 \mathrm{~mL}$,
Vascular $479 \mathrm{~mL}$, Thoracic $402 \mathrm{~mL}$ and Orto/Ortho-Spine $358 \mathrm{~mL}$, the $\%$ of cases requiring any blood transfusion by category was: CV 13.1\%, Vascular $13.33 \%$ and Thoracic//Ortho/Ortho-Spine $0 \%$ and number of cases using cell salvage were: CV 32, Orto/ Ortho-Spine 2 and Vascular/Thoracic 0 (Table 2). The economic impact of bleeding and transfusions ranged from $\$ 9,824$ to $\$ 29,296$ depending on the surgery and the hemostatic agent used.

\section{Discussion}

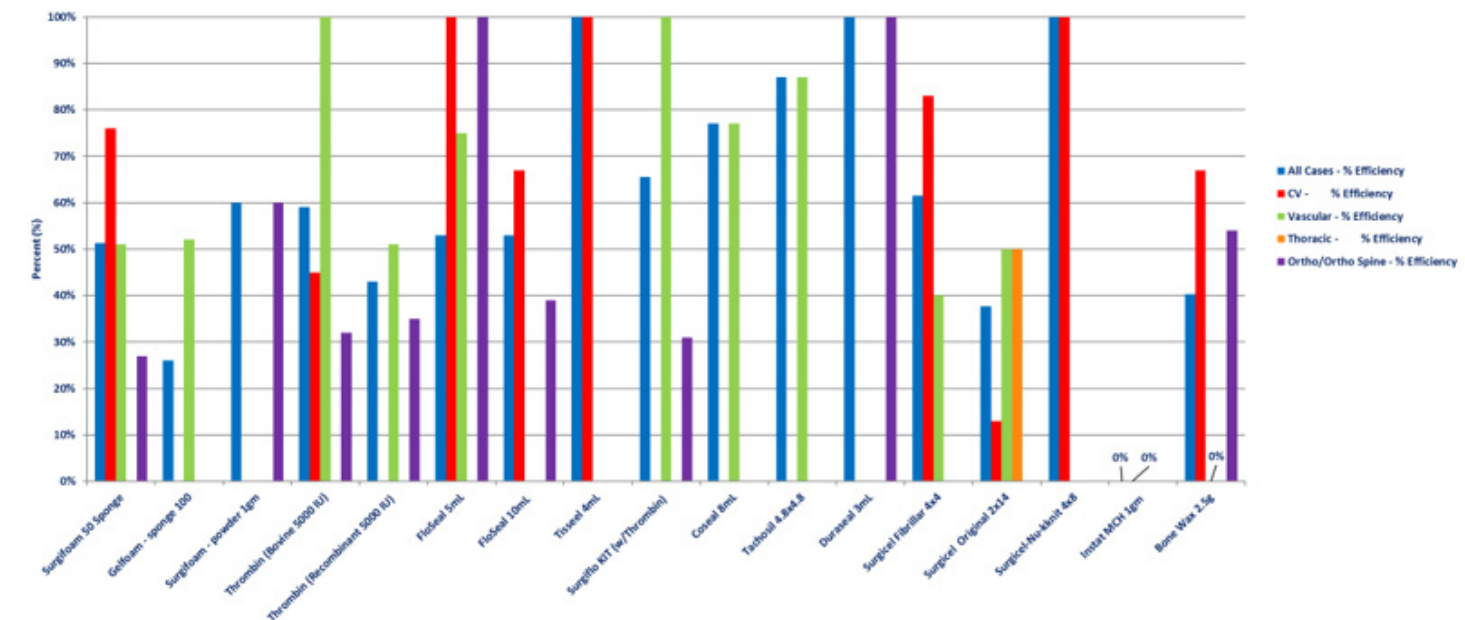

Source: Own elaboration with data from US Vital Edge Program, LUR Analyses retrospective analysis.

Figure 1: Utilization Efficiency by Specialty.

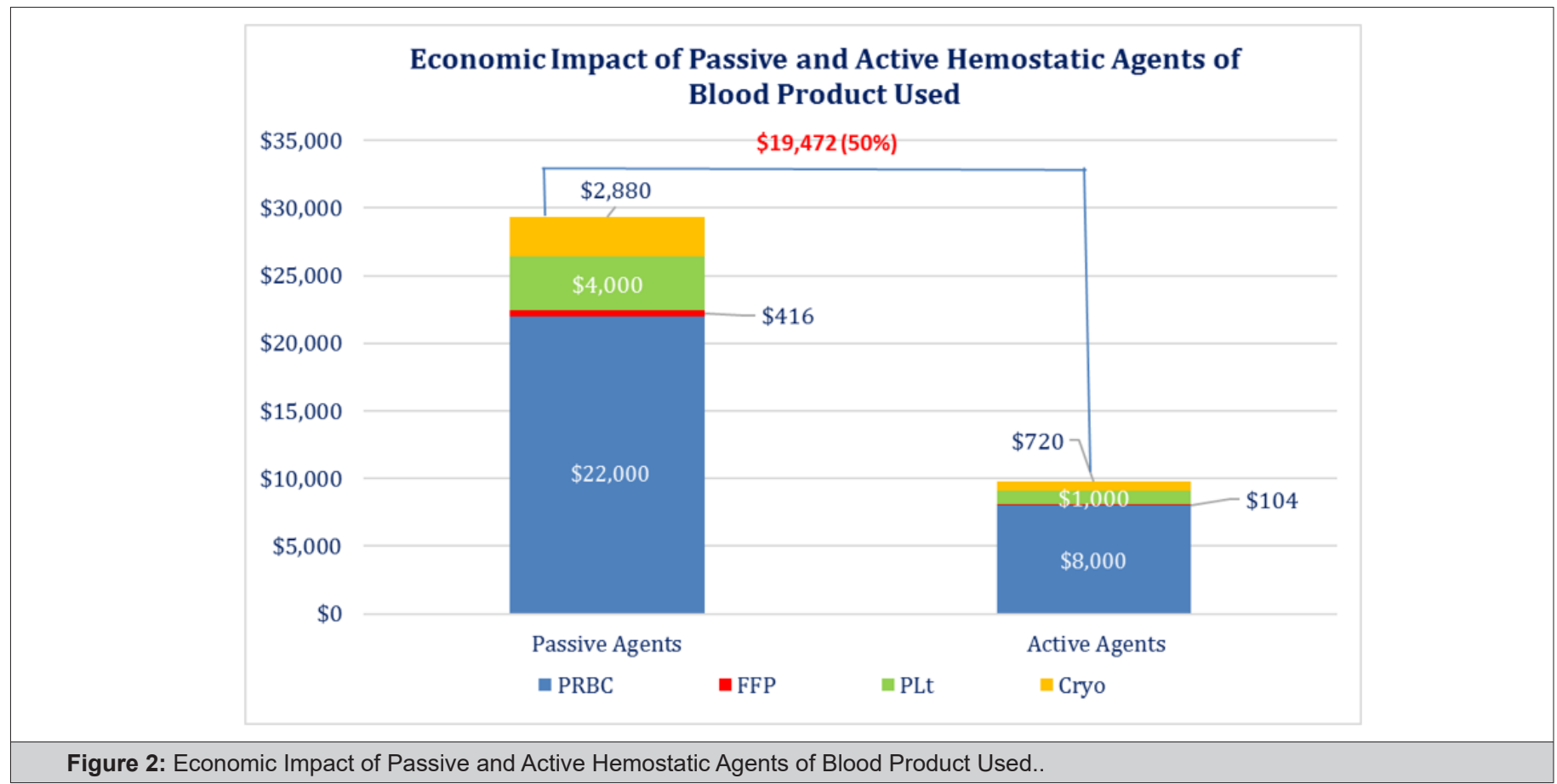

The complexity of surgical procedures in an aging population, where intraoperative blood loss can have devastating consequences, creates challenges for hospital' resources and, if not well managed, intraoperative bleeding in complex procedures can threaten patient near-term and long-term outcomes and expend unnecessary hospital resources. To address these dynamics and 
the challenges they present, associations, hospitals, surgeons, and policymakers must consider opportunities to drive new efficiencies in healthcare delivery to optimize patient outcomes. This is particularly important in high-risk surgeries. While there are several ways to create surgical efficiencies, and improve health outcomes, efficiencies that drive lower utilization of blood can indicate adoption of sustainable improvement initiatives across the hospital, resulting in better post-operative outcomes.

In a first step, established and respected institutions like the Society of Thoracic Surgeons, American Society of Anesthesiologists, The Joint Commission, the U.S. Department of Health and Human Services and others have recognized the importance of improving blood management and have proposed clinical guidelines to reduce the need for intraoperative transfusions through preoperative, intra-operative, and post-operative evidence-based recommendations. Beside the above strategies, it is important to choose the proper hemostatic agent (active product) to control/ stop the bleeding and reduce minor or major compactions related to bleeds. It is known that the use of active products can lead to improved intraoperative and postoperative transfusion requirements. Cost calculations reported here are hypothesis generating, do not represent a detailed or formal analysis of cost effectiveness and warrant further confirmation in dedicated economic studies. Nevertheless, based on the estimated $\$ 19,472$ $(50 \%)$ per patient cost saving associated with the use of hemostatic agents (active products), it seems that substantial reductions observed for some of the surgical requirements may have directly translated into cost savings (Figure 2).

\section{Limitations}

All economic models are a simplification of a complex healthcare situation and, hence, bear the limitations associated with a simple representation of the reality. Limitations of the analysis includes it is a retrospective analysis of an observational collected intraoperative data (72 Cardio Vascular/Thoracic \& 39 OrthoOrtho Spine surgeries), performed between August-December 2018, from different US Urban Hospitals database design, which is less robust than the conduct of prospective randomized trials and inherently associated with selection biases. In addition, the sample size analyzed Cardio Vascular/Thoracic and Ortho-Spine; if we include other surgical specialties the results may be due to chance. To avoid this, a randomized clinical trial with a larger number of patients and proper measuring on the clinical impact of inhibited blood loss during procedures and the complications derived from this should be carried out.

\section{Conclusion}

Table 3: Total Cost Savings of Blood Products Use.

\begin{tabular}{|c|c|c|c|c|}
\hline \multicolumn{5}{|c|}{ TOTAL COST SAVINGS OF BLOOD PRODUCT USE } \\
\hline Surgery Type & $\begin{array}{c}\text { Average EBL } \\
\text { (mL) Passive } \\
\text { Agents }\end{array}$ & $\begin{array}{l}\text { Average EBL } \\
\text { (mL) Active } \\
\text { Agents }\end{array}$ & $\begin{array}{c}\text { Economic Impact } \\
\text { of Blood Product } \\
\text { Used (Passive } \\
\text { Agents) }\end{array}$ & $\begin{array}{c}\text { Economic Impact } \\
\text { of Blood Product } \\
\text { Used (Active } \\
\text { Agents) }\end{array}$ \\
\hline CV & 363 & 268 & $\$ 21,296$ & $\$ 9,824$ \\
\hline Vascular & 350 & 129 & $\$ 8,000$ & \$0 \\
\hline Thoracic & 282 & 120 & \$o & $\$ 0$ \\
\hline Ortho/Ortho-Spine & 250 & 108 & \$o & \$o \\
\hline $\begin{array}{l}\text { Total Economic } \\
\text { Impact }\end{array}$ & 311 & 156 & $\$ 29,296$ & $\$ 9,824$ \\
\hline Total Savings & & & & $\$ 19,472$ \\
\hline
\end{tabular}

Surgical bleeds, leakages and transfusions can have substantial financial and medical outcome consequences, the prevention of which would be beneficial for institutions and patients. Reducing the need for blood transfusions by minimizing blood loss during surgery through the efficient use of appropriate hemostat agents could potentially eliminate the wasteful utilization of resources and likely improve patient post-surgical outcomes. The use of hemostatic agents (active products) resulted in a substantial Blood Product Use savings of $\$ 19,472$ (50\%) per patient. The use of hemostatic agents (active products) may result in effective and cost-beneficial as compared with hemostatic agents (passive products) (Table 3).

\section{Acknowledgement/Conflict of interest disclosures}

Authors (MGR, GFC and MAR) are employees of Baxter Healthcare, which are involved in the manufacture of a product examined in this analysis.

\section{References}

1. Shander A (2007) Financial and clinical outcome associated with surgical bleeding complications. Surgery 142(4): S20-S25.

2. Rady MY, Ryan T, Starr NJ (1998) Perioperative determinants of morbidity and mortality in elderly patients undergoing cardiac surgery. Crit Care Med 26(2): 225-235.

3. MichaelEStokes, Xin Ye, Manan Shah, Katie Mercaldi, MatthewW Reynolds et al. (2011) Impact of bleeding-related complications and/or blood 
product transfusions on hospital costs in inpatient surgical patients. BMC Health Services Research 11: 135.

4. Koren, RR (2017) Physicians lack of knowledge could be a possible reason for red blood cell transfusion overuse? Isr J Health Policy Res 6: 49.

5. De Frances C J, Lucas CA, Buie VC, Golosinskiy (2008) A 2006 National Hospital Discharge Survey. Natl Health Stat Rep 5: 1-20.

6. Von Ahsen N, Müller C, Serke S, Frei U, Eckardt K U (2001) Important role of nondiagnostic blood loss and blunted erythropoietic response in the anemia of medical intensive care patients. Crit. Care Med 29: S141-S150.

7. Tackett SM, Sugarman R, Kreuwel HT, Piedad Alvarez, Nasso G (2014) Hospital economic impact from hemostatic matrix usage in cardiac surgery. J Med Econ 17: 670-676.

8. Stokes ME, Ye X, Shah M, Katie Mercaldi, Matthew W Reynolds et al (2011) Impact of bleeding-related complications and/or blood product transfusions on hospital costs in inpatient surgical patients. BMC Health Serv Res 11: 135
9. Forbes JM, Anderson MD, Anderson GF, Bleecker GC, Rossi EC et al (1991) Blood transfusion costs: A multicenter study. Transfusion 31(4): 318-323.

10. Crémieux PY, Barrett B, Anderson K, Slavin MB (2000) Cost of outpatient blood transfusion in cancer patients. J Clin Oncol 18: 2755-2761.

11. (2018) Spotlight: Margin of Excellence-Blood Utilization Report.

12. (2017) The Joint Commission.

13. Chu M, Losenno K, Moore K, Berta D, Hewitt J et al. (2013) Blood conservation strategies reduce the need for transfusions in ascending and aortic arch surgery. Perfusion 28(4): 315-321

14. Borja Ribed Sánchez, Cristina González-Gaya, Sara Varea-Díaz, Carlos Corbacho Fabregat, Jaime Pérez Oteyza et al. (2018) Economic Analysis of the Reduction of Blood Transfusions during Surgical Procedures While Continuous Hemoglobin Monitoring Is Used. Sensors 18: 1367. 